\title{
Salvage brachytherapy for seminal vesicle recurrence after initial brachytherapy for prostate cancer: a case report
}

\author{
Shunta Hori ${ }^{1}$, Nobumichi Tanaka ${ }^{1 *}$, Isao Asakawa², Yosuke Morizawa' , Akihide Hirayama ${ }^{3}$, Masatoshi Hasegawa²,
} Noboru Konishi ${ }^{4}$ and Kiyohide Fujimoto ${ }^{1}$

\begin{abstract}
Background: To report the efficacy and safety of salvage brachytherapy for seminal vesicle recurrence after initial brachytherapy in a patient with prostate cancer. As far as we know, this is a first report of salvage brachytherapy for seminal vesicle recurrence in Japan.

Case presentation: A 70-year-old Japanese man with low-risk prostate cancer received low-dose-rate brachytherapy. Forty-two months after the seed implantation, he showed biochemical recurrence based on the nadir $+2 \mathrm{ng} / \mathrm{mL}$ definition. The prostate specific antigen (PSA) level was $5.11 \mathrm{ng} / \mathrm{mL}$ at 58 months after seed implantation. A saturation biopsy of the prostate showed no recurrence. Systemic screening also showed no distant metastases. However, T2-weighted magnetic resonance imaging (MRI) demonstrated a low intensity area at the base of the right seminal vesicle, which was strongly suggestive of recurrence. Sixty months after the initial therapy, a seminal vesicle biopsy confirmed recurrence with a Gleason score of $4+3$ before salvage brachytherapy was performed. The prescribed dose was $145 \mathrm{~Gy}$, the same as the dose of the initial therapy. One month later, the PSA level had rapidly declined to $0.898 \mathrm{ng} / \mathrm{mL}$ without androgen deprivation therapy. Ten months after the salvage brachytherapy, the PSA level reached $0.078 \mathrm{ng} / \mathrm{mL}$. No adverse events were seen during the follow-up period.
\end{abstract}

Conclusions: We experienced a patient who was successfully treated with salvage brachytherapy for seminal vesicle recurrence. Salvage brachytherapy is one of the promising therapeutic options for recurrence after initial brachytherapy.

Keywords: Salvage brachytherapy, Seminal vesicle recurrence, Prostate cancer

\section{Background}

Several salvage therapeutic options including radical prostatectomy, radiation therapy and androgen deprivation therapy can be considered for patients who show seminal vesicle recurrence after prostate brachytherapy. We experienced a patient who underwent salvage brachytherapy. This is the first report of a case of salvage brachytherapy for seminal vesicle recurrence after prostate brachytherapy in Japan, as far as we know.

\footnotetext{
*Correspondence: sendo@naramed-u.ac.jp

'Department of Urology, Nara Medical University, 840 Shijo-cho, Kashihara, Nara 634-8522, Japan

Full list of author information is available at the end of the article
}

\section{Case presentation}

A 70-year-old Japanese man underwent a medical checkup in March 2005, which showed an abnormal prostate specific antigen (PSA) value $(4.8 \mathrm{ng} / \mathrm{mL})$, and he consulted a urologist. He underwent needle biopsy of the prostate. The histopathological findings indicated an adenocarcinoma with a Gleason score of $3+3$. The number of positive cores was 4 out of 8 cores (right lobe: $1 / 4$, left lobe: $3 / 4$ ). The digital rectal examination showed no abnormal findings. The clinical stage was T1cN0M0. In 2006, he underwent a low-dose-rate brachytherapy (LDR-brachytherapy) using a preplanning method and modified peripheral loading at our hospital. The prescribed dose was $145 \mathrm{~Gy}$. Regarding dosimetric parameters using CT images 1 month after seed implantation, the minimal dose (Gy) received by $90 \%$ of the prostate gland (D90) was $183 \mathrm{~Gy}$, the 
percentage prostate volume that received $100 \%$ of the prescribed minimal peripheral dose (V100) was 98.5\%, and the rectal volume $(\mathrm{mL})$ that received $100 \%$ of the prescribed dose (R100) was $0.02 \mathrm{~mL}$. One year after seed implantation, the PSA level reached the nadir at $0.38 \mathrm{ng} / \mathrm{mL}$. The PSA level then increased gradually. Forty-two months later, PSA recurrence was confirmed based on the Phoenix definition (nadir $+2 \mathrm{ng} / \mathrm{mL}$ ). The patient was then observed without any salvage therapy because the dosimetric quality of the initial therapy was excellent and the possibility of PSA bounce was also considered. Fifty-eight months after seed implantation, the PSA level reached $5.11 \mathrm{ng} / \mathrm{mL}$. A prostate saturation biopsy demonstrated no obvious recurrence inside the prostate. Systemic screening by lung and abdominal CT and bone scan did not detect distant metastases. On the other hand, T2-weighted and dynamic MRI images demonstrated a low intensity area at the base of the right seminal vesicle, which was very suspicious of recurrence (Figure 1A,B,C). We proposed salvage prostatectomy, salvage brachytherapy or androgen deprivation therapy to the patient. Sixty months after the initial therapy, he underwent salvage brachytherapy after providing informed consent. The prescribed dose of the salvage therapy was $145 \mathrm{~Gy}$ as same as the initial therapy. We used a radioactive source of $0.33 \mathrm{mC} 1(13.1 \mathrm{MBq})$ for salvage brachytherapy (as same as initial brachytherapy). Prior to salvage brachytherapy, seminal vesicle biopsy was performed and the histological findings showed an adenocarcinoma with a Gleason score of $4+3$ at the base of the right seminal vesicle. The base of right seminal vesicle was set as the planning target volume (PTV) with $1 \mathrm{~cm}$ margin. Seven needles were inserted into the PTV, and 2 or 3 sources were implanted through each needle. Finally 20 sources were implanted by the real-time planning method
(Figure 2). Regarding dosimetric parameters using CT images 1 month after salvage brachytherapy, seminal vesicle D90 was 280 Gy, seminal vesicle V100 was $100 \%$, prostate D90 was 206.4 Gy, prostate V100 was $99.2 \%$, the R100 was $0.26 \mathrm{~mL}$, and the minimal dose (Gy) received by $30 \%$ of the urethra (UD30) was 285.7 Gy. The treatment time was 69 minutes. Since the PSA level rapidly decreased to $0.898 \mathrm{ng} / \mathrm{mL}$ by 1 month after seed implantation, he received no adjuvant therapy. Ten months after seed implantation, the PSA level reached $0.078 \mathrm{ng} / \mathrm{mL}$. No adverse events were seen during the follow-up period.

\section{Discussion}

When patients show PSA recurrence after LDRbrachytherapy, there are several options for salvage treatments such as androgen deprivation therapy, radical prostatectomy, radiation therapy, etc. A 70-year-old Japanese man has a life-expectancy of 15.1 years if no severe co-morbidities are present. We discussed the merits and demerits of each modality of salvage therapy with the patient. He finally chose salvage brachytherapy of the seminal vesicle. There have been several previous reports of salvage brachytherapy (Table 1). The oncological outcomes showed a biochemical recurrence-free rate of around $70 \%$. Some patients have showed genitourinary and gastrointestinal adverse events of grade 3 or greater, but the incidence rate was acceptable. Some reports involved prescription of a full dose (145 Gy), while the prescribed dose was reduced according to other reports [1-7]. There is not a standard prescribed dose and the pros and cons of adjuvant hormonal therapy are not established. As far as we know, there are two reports of salvage brachytherapy for seminal vesicle recurrence. Koutrouveil et al. reported 31 patients who showed recurrence after initial brachytherapy. Eleven of the 31
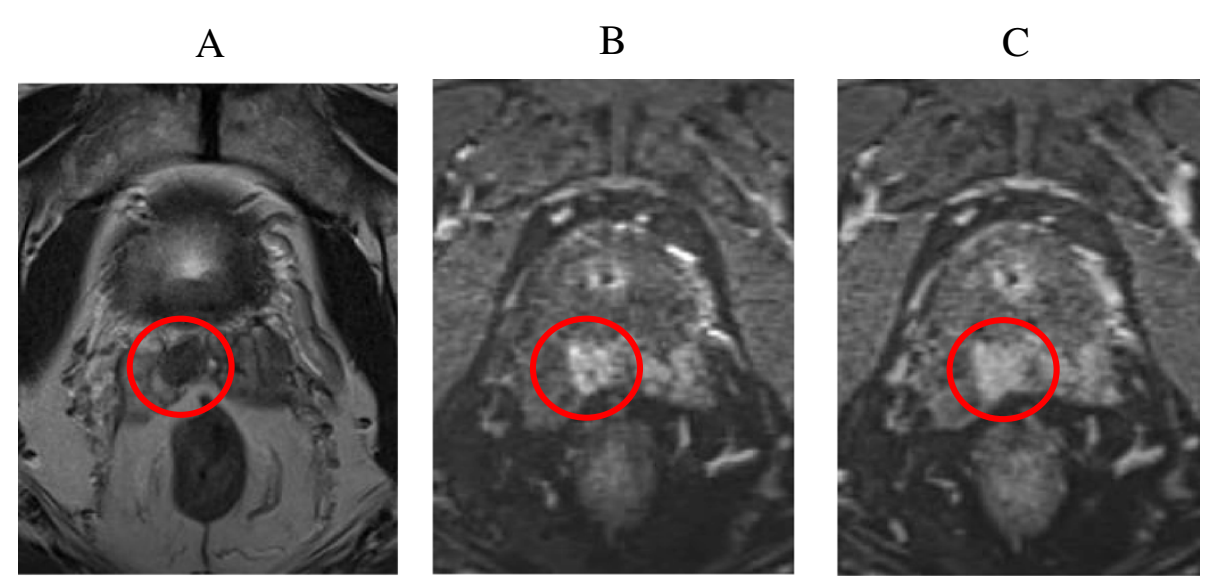

Figure $1 \mathrm{MRI}$ images of seminal vesicle recurrence. (A) T2-weighted image demonstrated a low-intensity area in the bottom of seminal vesicle. (B, C) Dynamic MRl also demonstrated a recurrence of seminal vesicle because of an intensive enhancement in early phase and a washed-out in late phase. 
A

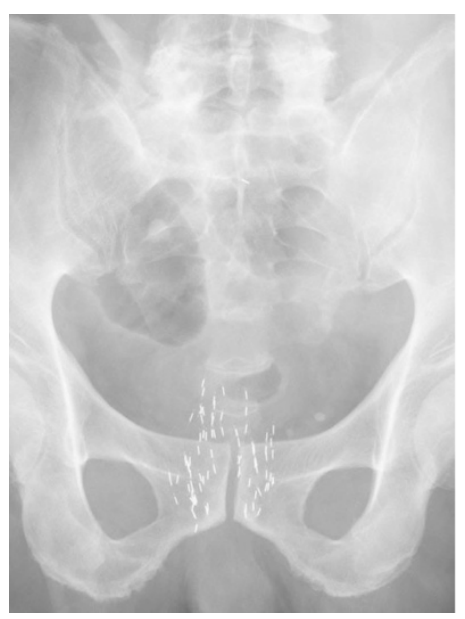

B

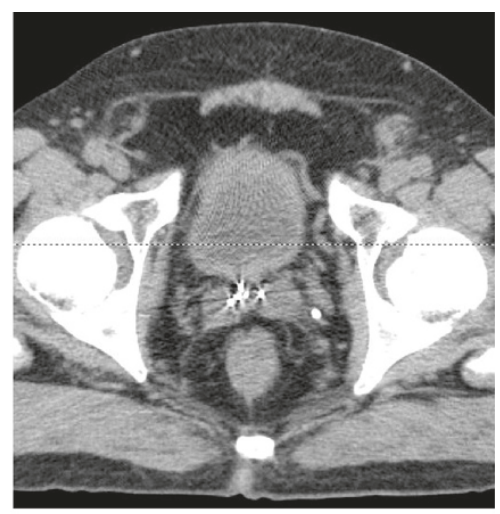

Figure 2 Pelvic X-ray and CT scan images after salvage brachytherapy. (A) Pelvic X-ray and (B) CT scan demonstrated the source at the bottom of seminal vesicle.

patients showed recurrence in the seminal vesicle, and 9 of these 11 patients showed no recurrence after salvage brachytherapy. They also showed that salvage brachytherapy was as useful as other salvage therapies because a high biochemical control rate (87\%) was achieved [1]. Burri et al. also reported the results of salvage brachytherapy in 37 patients. They concluded that a PSA level of less than 6 was a prognostic factor for salvage brachytherapy in multivariate analysis [2]. However, largescale studies on salvage brachytherapy have not been published. Therefore, patient selection, optimal prescribed dose, and use of adjuvant androgen deprivation therapy are still controversial. The excessive dose area of the seminal vesicle due to the small target volume may consequently lead to the problem that the urethral dose and the dose to the bottom of the prostate are apt to be high. It is also difficult to insert needles into the seminal vesicle because the distance from the perineum to the seminal vesicle is longer than to the prostate for seed implantation. With regard to the adverse events of salvage brachytherapy, the RTOG 0526 study is presently in progress and the results are awaited. Our patient shows a favorable outcome at present during the early stage after salvage therapy for seminal vesicle recurrence. Longer follow-up data involving more patients are needed to establish the usefulness of salvage brachytherapy, not only for seminal vesicle recurrence, but also for the primary focus.

\section{Conclusions}

We reported a case of salvage brachytherapy for seminal vesicle recurrence with a favorable outcome. It is

Table 1 Several previous reports of salvage brachytherapy

\begin{tabular}{|c|c|c|c|c|c|c|c|}
\hline Study year & $\mathbf{N}$ & Prior RT & Use of ADT & $\begin{array}{l}\text { Median } \\
\mathbf{f} / \mathbf{u}\end{array}$ & Outcome & Adverse event & Prescribed dose \\
\hline Koutrouveils* 2003 [1] & 31 & seed & $\begin{array}{l}\text { Neo } 3 \text { mos } \\
\text { except } 1\end{array}$ & 30 mos & 5 yr-bDFS: 87\% & Gl: G4 (2/31) & $\begin{array}{l}{ }^{125} \mathrm{l}: 100-144 \mathrm{~Gy} \\
{ }^{103} \mathrm{Pd}: 100-120 \mathrm{~Gy}\end{array}$ \\
\hline Wong 2006 [3] & 17 & EBRT & $\begin{array}{l}\text { Neo } 3 \text { mos: } 12 \\
\text { ad } 6 \text { mos: } 5\end{array}$ & 44 mos & 4 yr-bDFs: 75\% & GU:G4 (1/17) & ${ }^{125} \mid: 126$ Gy in principle \\
\hline Nguyen 2007 [4] & 25 & EBRT: 13 seed: 12 & none & 47 mos & 4 yr-bDFS: 70\% & GU: G3-4 13\% Gl: G3-4 30\% & ${ }^{125} \mid: 137$ Gy \\
\hline Lee 2007 [5] & 21 & EBRT & Concurrent-ad: 12 & 36 mos & 5 yr-bDFS: $38 \%$ & G3 or over: none & ${ }^{125} \mid: 90$ Gy \\
\hline Aaronson 2009 [6] & 24 & EBRT & Concurrent: $17 \%$ & 30 mos & 3 yr-bDFS: $89.5 \%$ & GU: G3 (1/24) & ${ }^{125} \mid: 144$ Gy (108 Gy) \\
\hline Moman 2010 [7] & 31 & EBRT: 20 seed: 11 & Neo 3 mos: 5 & 9.2 yrs & 5 yr-FFbF: 23\% & $\begin{array}{l}\text { lateGU: G3 (6/31) } \\
\text { lateGI: G3 (2/31) }\end{array}$ & ${ }^{125} \mid: 145$ Gy \\
\hline Burri* 2010 [2] & 37 & EBRT: 32 seed: 5 & $\mathrm{Neo}+$ ad 6 mos: 31 & 86 mos & $5 \mathrm{yr}-\mathrm{FFbF}: 64.5 \%$ & Gl: G4 (1/37) & ${ }^{125} \mid: 135$ Gy \\
\hline
\end{tabular}

*Report of brachytherapy for recurrence of seminal vesicle.

RT: Radiation Therapy, ADT: Androgen Deprivation Therapy, f/u: follow-up, EBRT : External Beam Radiation Therapy, Seed: Seed Implant (Brachytherapy), Neo: Neoadjuvant Therapy, Ad: Adjuvant Therapy, bDFS: biochemical Disease Free Survival, FFbF: Freedom From biochemical Failure, GI: Gastrointestinal adverse effect, GU: Genitourinary adverse effect, G: Grade. 
expected that salvage brachytherapy will play a significant role as a salvage therapeutic modality after radiation therapy including brachytherapy.

\section{Consent}

Written informed consent was obtained from the patient to publish this case report and any accompanying images. A copy of the written informed consent is available for review by the Editor-in-Chief of this journal.

\section{Abbreviations}

LDR-brachytherapy: Low-dose-rate brachytherapy; PSA: Prostate specific antigen; D90: Minimal dose (Gy) received by $90 \%$ of the prostate gland; V100: Percentage prostate volume that received $100 \%$ of the prescribed minimal peripheral dose; R100: Rectal volume $(\mathrm{mL})$ that received $100 \%$ of the prescribed dose; UD30: The minimal dose (Gy) received by $30 \%$ of the urethra; PTV: Planning target volume.

\section{Competing interests}

The authors declare that they have no competing interest.

\section{Authors' contributions}

All authors made substantial contributions to the acquisition and interpretation of data, critical revision of the manuscript for important intellectual content, and approved the final version for publication. KF, NK, $\mathrm{AH}$ and $\mathrm{MH}$ made substantial contributions to the conception and design of the study. NT, SH, YM and IA performed the treatment. All authors read and approved the final manuscript.

\section{Acknowledgements}

We kindly thank Miss Mariko Yoshimura for contribution to drafting this manuscript.

\section{Author details}

${ }^{1}$ Department of Urology, Nara Medical University, 840 Shijo-cho, Kashihara, Nara 634-8522, Japan. ${ }^{2}$ Department of Radiation Oncology, Nara Medical University, Nara, Japan. ${ }^{3}$ Department of Urology, Nara Hospital of Kinki University, Nara, Japan. ${ }^{4}$ Department of Pathology, Nara Medical University, Nara, Japan.

Received: 3 June 2014 Accepted: 13 October 2014

Published: 26 October 2014

\section{References}

1. Koutrouvelis P, Hendricks F, Lailas N, Gil-Montero G, Sehn J, Khawand N, Bondy $H$, Katz S: Salvage reimplantation in patient with local recurrent prostate carcinoma after brachytherapy with three dimensional computed tomography-guided permanent pararectal implant. Technol Cancer Res Treat 2003, 4:339-344.

2. Burri RJ, Stone NN, Unger P, Stock RG: Long-term outcome and toxicity of salvage brachytherapy for local failure after initial radiotherapy for prostate cancer. Int J Radiat Oncol Biol Phy 2010, 77:1338-1344.

3. Wong WW, Buskirk SJ, Schild SE, Prussak KA, Davis BJ: Combined prostate brachytherapy and short-term androgen deprivation therapy as salvage therapy for locally recurrent prostate cancer after external beam irradiation. J Urol 2006, 176:2020-2024.

4. Nguyen PL, Chen MH, D'Amico AV, Tempany CM, Steele GS, Albert M, Cormack RA, Carr-Locke DL, Bleday R, Suh WW: Magnetic resonance image-guided salvage brachytherapy after radiation in select men who initially presented with favorable-risk prostate cancer: a prospective phase 2 study. Cancer 2007, 110:1485-1492.

5. Lee HK, Adams MT, Motta J: Salvage prostate brachytherapy for localized prostate cancer failure after external beam radiation therapy. Brachytherapy 2008, 1:17-21.
6. Aaronson DS, Yamasaki I, Gottschalk A, Speight J, Hsu IC, Pickett B, Roach M 3rd, Shinohara K: Salvage permanent perineal radioactive-seed implantation for treating recurrence of localized prostate adenocarcinoma after external beam radiotherapy. BJU Int 2009, 104:600-604.

7. Moman MR, van der Poel HG, Battermann JJ, Moerland MA, van Vulpen M: Treatment outcome and toxicity after salvage 125-I implantation for prostate cancer recurrences after primary 125-I implantation and external beam radiotherapy. Brachytherapy 2010, 9:119-125.

doi:10.1186/1756-0500-7-760

Cite this article as: Hori et al:: Salvage brachytherapy for seminal vesicle recurrence after initial brachytherapy for prostate cancer: a case report. BMC Research Notes 2014 7:760.

\section{Submit your next manuscript to BioMed Central and take full advantage of:}

- Convenient online submission

- Thorough peer review

- No space constraints or color figure charges

- Immediate publication on acceptance

- Inclusion in PubMed, CAS, Scopus and Google Scholar

- Research which is freely available for redistribution 